\title{
Carlo Collodi, traducteur des contes de fées
}

\author{
François Bouchard \\ Université François Rabelais, Tours
}

Quand paraît en 1876, à Florence, le volume de I racconti delle fate voltati in italiano da Carlo Collodi, ${ }^{1}$ dans la « Biblioteca Scolastica » de Felice Paggi, éditeur spécialisé dans le secteur des publications pour la jeunesse, la renommée de Carlo Lorenzini est encore essentiellement liée à son activité de journaliste, qu'il a inaugurée en 1847 dans les pages de la revue milanaise L'Italia musicale. Et s'il a commencé à signer certains de ses articles du pseudonyme de Carlo Collodi dès 1856 , les deux premières œuvres narratives qu'il publie en volume le sont de son vrai nom sans qu'aucune ne révèle l'évidence d'un talent. ${ }^{2}$ C'est donc avec cette traduction que le futur auteur de Le avventure di Pinocchio aborde la littérature pour la jeunesse, pour laquelle il avait manifesté de l'intérêt par un article du 24 septembre 1875 consacré, dans Il Fanfulla, au premier roman d'Ida Bacini, Memorie di un pulcino, que celleci venait de publier chez Felice Paggi dans la même section, «Libri per letture » et la même collection, « Biblioteca scolastica » mais sans nom d'auteur afin que - selon le vœu de l'éditeur qu'elle rapporte dans son autobiographie — « beaucoup le croient dû à une plume masculine $\gg{ }^{3}$ Dès l'année suivante, Collodi publie Giannettino. Libro per i ragazzi (Paggi, 1877), premier de ses ouvrages pédagogiques destinés à un succès durable par le biais de l'institution scolaire. Et beaucoup d'autres lui font suite, liés à la figure du même Giannettino, soit par le biais d'un de ses camarades - Minuzzolo. Secondo libro di lettura (séguito al « Giannettino »), 1878 — , c'est bien à ces deux ouvrages, le roman et le recueil de nouvelles, que la traduction de I racconti delle fate conduit l'auteur par des voies détournées : en se colletant avec un corpus de textes

\footnotetext{
${ }^{1}$ I racconti delle fate voltati in italiano da Carlo Collodi. Firenze: Felice Paggi, 1876 ; 2e éd. Firenze : Felice Paggi, 1887. Désormais cité comme RF. Réédité dans le volume IV de l'Edizione Nazionale delle Opere : EN IV : Edizione Nazionale delle opere di Carlo Lorenzini Collodi. Pescia Firenze : Fondazione Nazionale Carlo Collodi-Giunti, 2015, vol. IV : I racconti delle fate voltati in italiano da Carlo Collodi, a cura e con introduzione di François Bouchard; Storie allegre, a cura e con introduzione di François Bouchard, prefazione di Guido Conti. Désormais cité comme EN IV.

${ }^{2}$ Un romanzo in vapore. Da Firenze a Livorno guida storico-umoristica di Carlo Lorenzini. Firenze : Giuseppe Mariani, 1856; I Misteri di Firenze Scene sociali di Carlo Lorenzini. Firenze : 1857, [date réelle : 1858]. Les deux textes s'inscrivent dans un genre relativement normé. Le premier a pour fonction de servir tout à la fois de guide et de lecture divertissante pour les usagers de la ligne de chemin de fer inaugurée entre Florence et Livourne en 1848. Le deuxième s'inscrit dans la vogue des Mystères qui a fait florès en Italie jusque tard dans le siècle. Et des deux volumes prévus des Misteri di Firenze, seul le premier voit le jour. Les deux premiers ouvrages de Lorenzini-Collodi sont rééditées dans: Edizione Nazionale delle Opere di Carlo Lorenzini Collodi. Vol. I. Firenze : Fondazione Nazionale Carlo Collodi-Giunti, 2010 : Carlo Lorenzini, Un romanzo in vapore. Da Firenze a Livorno, a cura di Roberto Randaccio, prefazione di Michèle Merger, introduzione di Elvio Guagnini; I Misteri di Firenze, a cura e con introduzione di Roberto Randaccio, prefazione di Andrea Camilleri. Désormais cité comme EN I.

${ }^{3}$ « Basti il dire che Felice Paggi, editore del mio primo libro, mi consigliò a non firmarlo o [...] a non scrivere per intero il mio nome di donna. — Basterà l'iniziale — aggiunse — così molti lo crederanno dovuto a penna maschile !» (Baccini, 17, nota 12).
} 
empruntés aux contes de fées français produit entre la fin du XVIIe et la première moitié du XVIIIe siècle.

Le terme même de « racconto delle fate » semble attesté pour la première fois en italien en 1728, avec la publication à Venise de la traduction de deux recueils de la Comtesse de Murat, Les Contes de fées (1698) et Les Nouveaux contes des fées (1698), sous le titre de: I racconti delle fate et I nuovi racconti delle fate (Venezia, presso Sebastiano Coleti, 1728). Et il vient s'ajouter aux catégories utilisées à partir du XVIe siècle pour désigner le conte merveilleux dans la littérature italienne : la « favola », qui est le terme avec lequel Giovan Francesco Straparola nomme chacune des nouvelles - même réalistes - qui composent le recueil de Le piacevoli notti (1550-1553); le « racconto » ou, en napolitain, " cunto », auquel recourent aussi bien Giambattista Basile dans Lo cunto de li cunti overo lo trattenemiento de' peccerille (1634-1636) que Pompeo Sarnelli dans la Posilecheata (1684); la « novella » qui sert à qualifier tantôt le court récit oral issu de la culture populaire que les folkloristes italiens — ou demopsicologi — s'emploient à recueillir et à transcrire avec une fidélité variable dans la deuxième moitié du XIXe siècle, tels Vittorio Imbriani avec $L a$ novellaja fiorentina (1877) et Gherardo Nerucci et ses Sessanta novelle popolari montalesi (1880), tantôt des textes ayant donné lieu à une élaboration personnelle, dans le cadre d'un imaginaire emprunté à cette même tradition : comme Le tre maruzze. Novella trojana da non mostrarsi alle signore (1875) de Vittorio Imbriani ; et enfin la « fiaba » qui s'impose progressivement de façon hégémonique tant pour la production populaire (Fiabe mantovane raccolte da Isaia Visentini, 1879) que pour l'œuvre d'invention individuelle à destination d'un public d'enfants ou d'adultes, comme C'era una volta... Fiabe (1882) de Luigi Capuana. Toutefois, les deux termes, fiaba et novella, continuent à coexister pendant tout le XIXe siècle.

Ainsi que l'a montré récemment Veronica Bonanni ( $L$ 'oiseau et $I$ racconti), Collodi ne traduit pas un corpus de textes qu'il aurait préalablement choisis $^{4}$ - mais une anthologie préexistante, les Contes de fées tirés de Claude (sic) Perrault, de Mme d'Aulnoy et de Mme Leprince de Beaumont, parue pour la première fois en 1853 dans la section rose de la Bibliothèque des Chemins de fer de Hachette qui deviendra par la suite la Bibliothèque rose illustrée, et destinée à un public de moins de quatorze ans selon les termes de l'auteur anonyme de la préface. ${ }^{5}$ Or, le recueil Hachette se compose de contes choisis en fonction de leur lisibilité ou de leur acceptabilité par le public visé. Aucune

\footnotetext{
${ }^{4}$ Contrairement à ce qu'a avancé en 1979 Fernando Tempesti, dans sa note à une réédition de cette traduction qui porte le titre fantaisiste de I racconti di Mamma Oca : «Il Collodi, com'è noto, tradusse per l'editore Paggi I racconti delle fate nel 1875 avendo molto probabilmente sott'occhio - e questo è meno noto - il testo del Cabinet des fées» [" On sait que Collodi a traduit per l'éditeur Paggi I racconti delle fate en 1875 en ayant probablement sous les yeux cela, on le sait moins - le texte du Cabinet des fées »], Fernando Tempesti. «Nota all'edizione italiana. "I racconti di Mamma Oca. Le favole di Perrault seguite da favole di Madame d'Aulnoy e di Madame Leprince de Beaumont, traduzione di Carlo Collodi. Milano : Feltrinelli, 1979, 25. La grande entreprise éditoriale du Cabinet des fées, menée à bien par Charles-Joseph de Mayer, compte un ensemble de quarante et un volumes publiés à Amsterdam entre 1785 et 1789.

5 « Deux femmes, Mme d'Aulnoy et Mme Leprince de Beaumont, partagent avec lui [Perrault] le privilège d'amuser tous les gens d'esprit qui n'ont pas encore quatorze ans ». "Préface. » Contes de fées tirés de Claude (sic) Perrault, de Mme d'Aulnoy et de Mme Leprince de Beaumont. Paris : Librairie de L. Hachette, 1860, II. Désormais cité comme Hachette 1860.
} 
préoccupation philologique ne préside au choix des textes. Le recueil original de Charles Perrault, les Histoires ou Contes du temps passé avec des moralités (1697), n'est pas reproduit dans son intégralité et l'ordre des textes y est bousculé de façon arbitraire. ${ }^{6}$ Le conte intitulé Les Fées est omis pour les raisons mêmes qui font de lui « une méditation mondaine sur les conditions de possibilité de la vie en société » (Gheeraert 432), peu adaptée à un public jeune. En revanche, la version apocryphe en prose de Peau d'âne, publiée par l'éditeur Lamy en 1781, est jointe sans aucun commentaire. ${ }^{7}$ Viennent s'ajouter quatre contes de Mme d'Aulnoy : La Belle aux cheveux d'or et L'Oiseau bleu, extraits des Contes de fées de 1697; La Chatte blanche et La Biche au bois qui proviennent du recueil suivant, Contes nouveaux ou les Fées à la mode ${ }^{8}$ (1698), où ils apparaissent en ordre contraire, dans les tomes II et I de l'ouvrage. Enfin, deux contes de Mme Leprince de Beaumont concluent le recueil, Le Prince Chéri et La Belle et la Bête, l'un et l'autre tirés du Magasin des enfants, ou dialogue entre une sage gouvernante et plusieurs de ses élèves de la première distinction (Londres, 1757), qui appartiennent à une autre saison de l'histoire du conte de fées et à un autre contexte culturel: le récit didactique destiné à transmettre un savoir moral et comportemental à un public enfantin.

Cette hétérogénéité des auteurs et de leurs œuvres est atténuée dans l'édition française par la présence d'un appareil iconographique, d'abord de 40 illustrations, qui passent à 65 à partir de la réédition de 1866, où sont rassemblés des illustrateurs de qualité inégale, tels Bertall, Beaucé, Jean-Achille Pouget, Predhom — dont la répartition régulière dans tout le volume contribue à lui conférer une cohérence graphique qui se reflète sur le contenu. En revanche, la préface anonyme se borne à donner quelques indications biobibliographiques sur les trois auteurs, relevés de jugements à l'emporte-pièce, sans pourtant citer in extenso le titre du recueil de Perrault. ${ }^{9}$

Avant la traduction de Collodi, le même volume avait donné lieu à une première traduction italienne par les soins d'un autre écrivain, Cesare Donati, selon des critères essentiellement conservatifs. ${ }^{10}$ Cette édition Jouhaud gardait le paratexte de l'originale, le même appareil iconographique postérieur à 1866, la même disposition des contes qui, dans le corps du recueil Hachette, sont précédés du nom de chacun des trois auteurs, et la même préface à laquelle le traducteur se bornait à ajouter une déclaration d'intention relative à son travail : « pour autant qu'il lui était possible, le traducteur a cherché à ce que la nouvelle

\footnotetext{
${ }^{6}$ À l'ordre original des Histoires ou Contes du temps passé - la dédicace À Mademoiselle, La Belle au bois dormant, Le Petit Chaperon rouge, La Barbe bleue, Le Maître chat, ou le Chat botté, Les Fées (omis), Cendrillon ou la petite pantoufle de verre, Riquet à la houppe, et Le Petit Poucet -, lequel a une cohérence interne liée à l'oscillation entre les deux genres, l'histoire et le conte, et qui se clôt sur le retour aux réalités du siècle à travers la référence à la guerre de la Ligue d'Augsbourg et au trafic des offices, se substitue le suivant dans le volume Hachette des Contes de fées : La Barbe Bleue, Le Petit Chaperon Rouge, La Belle au Bois dormant, Le Chat Botté, Cendrillon, Riquet à la Houppe, Le Petit Poucet, Peau d'Âne.

${ }^{7}$ Alors que la version originale en vers appartient à un précédent recueil de Perrault, intitulé Grisélidis, nouvelle. Avec le conte de Peau d'Âne et celui des Souhaits ridicules (Paris, 1694).

${ }^{8}$ Madame d'Aulnoy, Conte nouveaux ou les Fées à la mode. Edition critique établie par Nadine Jasmin. Paris : Honoré Champion, 2008. Désormais cité comme CN.

${ }^{9}$ «Les Contes de Perrault sont écrits avec une simplicité élégante et naïve, qui en fait une lecture véritablement attrayante ». Préface. Hachette 1860, I.

${ }^{10}$ I racconti delle fate tratti da Perrault, d'Aulnoy e Leprince de Beaumont, versione italiana di Cesare Donati adorna di 63 vignette per Bertall, Beaucé, ec.. Firenze : Stefano Jouhaud editore, 1867. Désormais cité comme Donati.
} 
forme n'intervienne pas au détriment de la belle simplicité qui constitue le principal ornement de l'original, ni qu'elle serve à dissimuler les qualités pour lesquelles celui-ci est célébré » (Donati Prefazione 3). ${ }^{11}$

Il en est tout autrement de la version de Collodi, laquelle refonde le paratexte et en modifie la fonctionnalité. Les trois auteurs disparaissent de la page de titre, sur laquelle le nom du traducteur s'inscrit dans une position hégémonique qui de fait l'érige en unique auteur du recueil. ${ }^{12}$ Tandis que le frontispice s'orne d'une illustration d'Enrico Mazzanti, avec qui collaborera Collodi pour l'ensemble de sa production publiée chez Paggi, et notamment $L e$ avventure di Pinocchio ; et celle-ci est le premier des six hors-textes qui composent tout l'appareil iconographique de cette édition. L'illustration représente la Barbe Bleue vêtu à la mode entre XVIe et XVIIe siècle d'une collerette et d'un pourpoint qui, l'air sombre, un poignard dans la main droite, désigne le sol de l'index gauche à l'adresse de sa femme qui, agenouillée devant lui, joint les mains en une pose suppliante. Délimité par une draperie sur la droite, qui masque en partie une fenêtre ouverte dans le mur du fond, l'espace évoque l'avant-scène d'un théâtre auquel renvoie également la barbe sombre du personnage, qui est celle du tyran dans le théâtre populaire italien, joué encore au XIXe siècle par les acteurs de chair et d'os comme par les marionnettes. ${ }^{13} \mathrm{Et}$ les traits de la femme de la Barbe Bleue, étrangement enfantins et inaboutis dans l'âge, contribuent à faire de celui qui la menace une figure conventionnelle, assignée à un rôle connu du tout-venant, bien éloignée de la Barbe Bleue que représente Bertall dans l'édition Hachette (Hachette 1860, 10), quand, le visage défiguré par un rictus satanique, il brandit un sabre et s'apprête à décapiter sa femme dont il maintient la tête baissée en empoignant ses longs cheveux bruns. La légende sous l'illustration renvoie à la page 13 du volume : à l'instant fatal où le mari dit à sa femme : «Sono inutili i piagnistei ...; bisogna morire » (13), à savoir : "Cela ne sert de rien ... ; il faut mourir » (9). Comme les autres, l'illustration de Mazzanti tend à euphémiser la représentation de la violence mise en scène dans les contes, ainsi que leur dimension inquiétante ou aberrante : comme le tête-à-tête entre la Belle et la Bête (RF 1876, hors texte 298-299) qui réinterprète une illustration de Bertall (364) en donnant au monstre une allure reconnaissable, en partie anthropomorphique, et en l'affublant d'un sourire bonhomme là où le Français avait représenté un être aux membres diaphanes dont l'apparence ne le rattachait à aucune créature identifiable.

Passée la page de titre, l'ouvrage s'ouvre sur un avertissement signé du traducteur où, faute de présenter explicitement ses choix méthodologiques, Collodi prend quelques précautions destinées à devancer les jugements qui

\footnotetext{
${ }^{11}$ « ... il traduttore cercò per quanto era in lui, che la nuova veste non riuscisse a scapito della bella simplicità dell'originale, né servisse a nascondere i pregi per cui va celebrato.»

${ }^{12}$ I / Racconti delle fate / voltati in italiano / da / C. Collodi / Firenze / Felice Paggi LibraioEditore / Via del Proconsolo / 1876.

${ }^{13}$ Et nombreuses sont les traces de ce type théâtral dans l'œuvre de Collodi. Dans Giannettino. Libro per i ragazzi (Firenze : Bemporad, 1892, 20), lorsque le jeune garçon reçoit en cadeau un boîte de marionnettes de Nuremberg où trône la figure du Tyran qui « effrayait rien qu'à le regarder » avec sa « grande barbe couleur vert bouteille » [ " Il Tiranno poi metteva paura soltanto a guardarlo !... / Aveva un gran barbone color verde bottiglia... »], comme dans $L a$ lanterna magica di Giannettino. Libro per i giovanetti (Firenze : Bemporad, 1902, 142) où Giannettino demande aux élèves de son oncle, le maître d'école : « Est-ce que j'ai l'air d'un croquemitaine comme les tyrans des tragédies » [ « ho forse l'aria di mangiabambini come i tiranni delle tragedie ?»]?
} 
pourraient être portés sur son travail. Et c'est la fidélité au texte original qu'il revendique en premier lieu ( « je m'employai autant qu'il me l'était possible de rester fidèle au texte français $»)$, laquelle exclut la paraphrase trop libre qui ferait fi de l'original (« Les paraphraser à ma volonté m'aurait semblé un demisacrilège »). ${ }^{14}$ Mais dans cette adhésion autoproclamée à l'original, intervient la marge de liberté que se concède le traducteur, les " variantes très légères » tant dans le choix du lexique (" sia di vocabolo ») que dans la structuration phrastique («sia di andatura di periodo ») et le registre stylistique (« sia di modi di dire »), qu'il s'empresse de révéler. Loin donc du parti pris d'extrême humilité que proclame Cesare Donati, qui se traduit par une fidélité méticuleuse à la lettre du texte original.

Enfin, le volume commence par « Barba-blu », sans que soit précisé le nom de l'auteur de la première série de contes. Pas de page donc annonçant « Contes de Perrault », "Contes de Madame d'Aulnoy », " Contes de Madame Leprince de Beaumont» comme dans l'édition Hachette : juste une anthologie de textes qui tendent à s'indifférencier pour le lecteur n'ayant pas une conscience immédiate de passer d'un auteur à un autre. Pour avoir cet élément d'information, il lui faut consulter la table des matières en fin de volume, dans laquelle ils ont été refoulés. Et par ce biais, le traducteur s'érige en figure auctoriale, car seul détenteur d'une autorité dont sont en partie dépossédés les auteurs, relégués dans les marges de l'œuvre, laquelle acquiert de ce fait une cohérence presque factice que la traduction accentue. En effet, quand même Les Contes de fées rassemblent trois auteurs aux poétiques et aux styles différents, Collodi s'empare de l'ensemble comme d'un tout sur lequel sa traduction passe un vernis unificateur qui, sans abolir les différences, confère aux trois ensembles de textes un air de déjà vu, une familiarité insolite que les œuvres originales n'avaient pas.

À cette dimension refondatrice de la traduction, il convient d'ajouter ce qui constitue l'une des caractéristiques des livres publiés dans la Bibliothèque des Chemins de fer: des textes parfois expurgés ou revus, souvent publiés dans des versions négligées, voire fautives. « D'ailleurs, le respect du texte n'est pas une préoccupation de l'éditeur », précise Elisabeth Parinet (104); et une preuve criante en est donnée par l'erreur qui entache le prénom de Perrault dans le titre de l'anthologie (Contes de fées tirés de Claude (sic) Perrault, de Mme d'Aulnoy et de Mme Leprince de Beaumont), laquelle est reproduite sans discontinuer jusqu'en 1950, y compris après le passage de l'ouvrage dans la Bibliothèque rose illustrée. Et certaines de ces erreurs altérant le sens ou le brouillant, la traduction s'en ressent inévitablement.

Ainsi en est-il de la forme lacunaire des titres de certains contes comme Le Chat botté (Hachette 1860, 36) - au lieu de Le Maître chat, ou le Chat botté - qui produit Il Gatto cogli stivali (RF, EN IV, 59); - tout comme Cendrillon (45) au lieu de Cendrillon ou la petite pantoufle de verre (version Collodi : Cenerentola, EN IV, 64). Ou de phrases et d'expressions fautives comme, par exemple, dans Le Petit Chaperon rouge, la leçon : « sa mère ayant fait et cuit des galettes » (12), au lieu de : « sa mère ayant cuit et fait des galettes » (Contes

\footnotetext{
${ }^{14}$ AVVERTENZA / Nel voltare in italiano i Racconti delle Fate, m'ingegnai, per quanto era in me, di serbarmi fedele al testo francese. Parafrasarli a mano libero, mi sarebbe parso un mezzo sacrilegio » ( RF 1876, 5 ; EN III, 30).
} 
196), où le verbe cuire a le sens absolu de cuire le pain ${ }^{15}$; ce qui aboutit à une traduction qui ratifie l'omission du pain pour ne plus mentionner que les galettes qu'on sort du four, alors que celles-ci ne sont qu'un complément ajouté ponctuellement à la fournée hebdomadaire (« sua madre, avendo cavate di forno alcune stiacciate », EN IV, 47). Dans L'Oiseau bleu de Madame d'Aulnoy, la transformation fautive du subjonctif imparfait de deux verbes («l'on fut tout étonné qu'il l'épousât, et que le noir se changeât en vert », Conte des fées ${ }^{16} 158$ ) en passé simple, tout en induisant une construction agrammaticale (« l'on fut tout étonné qu'il l'épousa, et que le noir se changea en vert », Hachette 1860, 142), brouille le sens de la phrase et donne ainsi lieu, dans la traduction, à l'introduction d'un décalage temporel entre mariage célébré et présenté comme un fait accompli, et surprise qu'il suscite à la cour : « tutti cascarono dalle nuvole, nel sentire che il Re l'aveva sposata, e che il nero s'era cambiato in verde » (EN IV, 120).

De plus, certaines de ces erreurs naissent, dans le volume des Contes de fées de Hachette, à l'occasion d'une des nombreuses réimpressions. Ainsi, dans l'édition 1860, on trouve dans La Belle et la Bête de Madame Leprince de Beaumont, que, après des revers de fortune, " le marchand et ses trois fils s'occupèrent à labourer la terre " : une leçon parfaitement fidèle au texte original ${ }^{17}$ que Cesare Donati traduit sans surprise par : « il mercante e i suoi tre figliuoli s'occuparono a lavorare la terra » (Donati, 385). En revanche, à partir de la réimpresion de 1871, apparaît la leçon fautive : « le marchand et ses trois filles s'occupèrent à labourer la terre » (Hachette 1871, 389) que Collodi rend scrupuleusement dans sa version par : «il mercante e le sue tre figlie si dettero subito a lavorare i campi » (EN IV, 242).

De surcroît, la traduction de Collodi tend à accentuer l'enracinement oral des textes traduits, lequel est une fiction fondatrice auquel tous les auteurs sacrifient selon des modalités différentes : chez Madame d'Aulnoy, nombre de contes sont lus à haute voix en société dans le cadre discontinu où sont enchâssés les Contes nouveaux ou les Fées à la mode, encore que cette lecture se fasse à partir d'une trace écrite qui est le fruit d'un jeu social ; chez Madame Leprince de Beaumont, les contes s'insèrent dans un dialogue serré entre une gouvernante, Mademoiselle Bonne, et ses élèves, pour y servir de divertissement mais aussi d'illustration aux leçons imparties dans le Magasin des enfants, ou dialogue entre une sage gouvernante et plusieurs de ses élèves de la première distinction, que l'auteur fit imprimer à Londres en 1757, soit six décennies après Madame d'Aulnoy. Enfin, chez Perrault, cette origine orale est rappelée tant dans l'édition de 1697 des Histoires ou Contes du temps passé avec des moralités (1697), que dans le manuscrit de 1695 conservé à la Perpoint Morgan Library à New York, par le frontispice où est représentée la nourrice, la « mie », servante et femme du peuple, qui, la quenouille à la main, file devant l'âtre pendant que l'écoutent deux enfants, fille et garçon, et un jeune homme assis sur une chaise le dos à la flamme, tous les trois vêtus d'une manière qui

\footnotetext{
15 « [Cuire] se met quelquefois absolument pour cuire du pain. » Dictionnaire de l'Académie française. $1^{\mathrm{e}}$ édition, 1694.

${ }^{16}$ Madame d'Aulnoy, Contes des fées, édition critique établie par Nadine Jasmin. Paris : Honoré Champion, 2008. Désormais cité comme CdF.

${ }^{17}$ Voir Madame de Villeneuve, La Jeune Américaine et les Contes marins (La Belle et la Bête) Les Belles solitaires, avec Madame Leprince de Beaumont, Magasin des enfants (La Belle et la Bête), édition critique établie par Élisa Biancardi. Paris : Honoré Champion, 2008, 1018.
} 
reflète leur appartenance sociale aisée. L'espace domestique est circonscrit par la lueur du feu dans l'âtre et la lueur d'une bougie posée sur le manteau de la cheminée ainsi que, à l'arrière-plan, par un porte close où est clouée une plaque avec cette inscription : CONTES / DE MA / MÈRE LOYE (sic), savoir ce que la première édition du Dictionnaire de l'Académie française (1694) définit en ces termes: "Le vulgaire appelle, conte au vieux loup, conte de vieille, conte de ma Mère l'Oye, conte de la cigogne, conte à la cigogne, conte de peau d'asne, conte à dormir debout ..., des fables ridicules telles que sont celles dont les vieilles gens entretiennent et amusent les enfants. »

Mais cette origine mythique est rendue, entre autres, chez Perrault par l'usage de formes archaïques censées témoigner d'un enracinement dans un vernaculaire nécessairement plus conservateur que la langue en usage dans les cercles de la bourgeoisie parisienne à laquelle appartient Perrault. D'où certaines de ces formules devenues d'autant plus populaires que le sens en est opaque, comme dans La Barbe Bleue, l'échange entre sa femme et sa sœur : " Anne, ma sœur Anne, ne vois-tu rien venir ? ... - Je ne vois rien que le soleil qui poudroie, et l'herbe qui verdoie » (Hachette 1860, 8). Quand " verdoyer » est déjà noté comme vieux par le Dictionnaire de l'Académie française, tandis que « poudroyer » n'est même plus attesté par les lexicographes de l'époque. Ce que Collodi traduit par : « Non vedo altro che il sole che fiammeggia e l'erba che verdeggia » $(E N$ IV, 44$)$, qui joue de la même allitération à partir de verbes inscrits dans un usage commun et dans un registre littéraire. Ou bien la recommandation bien connue de la mère-grand à qui frappe à sa porte : «Tire la chevillette, la bobinette cherra » (Hachette 1860, 15), où non seulement « chevillette » et « bobinette » ne sont pas attestées par les dictionnaires du temps ni associées aux arts de la serrurerie, mais le futur du verbe choir est noté par Furetière, qui le regrette, comme désuet. Une complexité dont la traduction de Collodi ne rend pas compte, car il fait le choix de la sacrifier à la simplicité immédiate de la langue parlée, réduisant le dispositif au seul pêne (stanghetta) : « Tira la stanghetta, e la porta si aprirà » (EN IV, 48).

En revanche, Collodi utilise un procédé semblable dans sa traduction lorsqu'il ancre le discours des personnages dans un terroir linguistique vernaculaire, marqué par une forte identité toscane, voire florentine. Tel le Petit Chaperon rouge qui, à la question du loup si la mère-grand habite loin ou non, lui répond : "La sta laggiù » (47), en recourant au pronom sujet la, toscan populaire et littéraire, aphérèse de ella. Tout comme le loup travesti en mèregrand, devant répondre aux questions du Petit Poucet jusqu'à la catastrophe

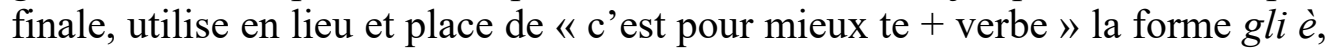
où de nouveau intervient un pronom sujet produit d'une aphérèse, qu'Ornella Castellani Pollidori définit comme étant « idiomatique (mais largement utilisé en littérature, comme d'autres traits typiquement florentins). ${ }^{18}$ Et la succession des réponses du loup (« Gli è per abbracciarti meglio »; « Gli è per correr meglio »; « Gli è per sentirci meglio » se conclut par le dramatique et attendu : « Gli è per mangiarti meglio » (49). La même forme reparaît dans la bouche de Perroquet, l'un des compagnons de la Princesse recluse dans la tour avant sa métamorphose en Chatte blanche dans le conte homonyme de Madame d'Aulnoy, quand l'oiseau vert vient annoncer à la jeune fille l'identité du

18 Ornella Castellani Pollidori. Introduzione. Le avventure di Pinocchio de Carlo Collodi. Edizione critica a cura di Ornella Castellani Pollidori. Pescia : Fondazione Nazionale Carlo Collodi, 1983, LXXX. 
prétendant auquel les fées qui la tiennent prisonnière entendent la marier. « Gli è un fagotto di panni sudici da far paura », déclare Papagallo (185) dans la traduction de Collodi, pour " c'est un magot qui fait peur » (Hachette 1860, 250) : où, à la forme vernaculaire toscane gli è dont use abondamment le traducteur, vient se surajouter fagotto di panni sudici pour rendre magot qu'il était aisé de traduire par scarabocchio ou stronco comme ailleurs dans I racconti delle fate afin de rendre son sens d' " homme laid». Mais Collodi lui substitue un terme injurieux, fagotto, qui est la personne habillée gauchement (« persona goffa, o goffamente vestita $»){ }^{19}$ et une insulte grave qui devient encore plus offensante avec le syntagme complet. ${ }^{20}$ Ainsi passe-t-on du qualificatif dépréciateur à l'insulte explicite, par un processus d'amplification expressive qui s'éloigne sciemment de la concision et du niveau de langue classique présent dans le texte de Madame d'Aulnoy pour virer au qualificatif plébéien. Toutefois, le traducteur ne renonce pas à la seconde occurrence de magot, celle de grand singe, qu'il prend en considération pour la réinjecter dans le texte à la fin du paragraphe suivant, quand il traduit « Je me résolvais plutôt à mourir qu'à l'épouser » (250) par : " Piuttosto che sposare quello scimmiotto mi veniva voglia di lasciarmi morire » (EN IV, 185), en profitant du pronom complément pour introduire la référence simiesque (sciommiotto).

Mais qu'elle se double ou non d'une trivialisation du niveau de langue, l'amplification expressive tend à rattacher le texte à des normes discursives qui appartiennent à la langue parlée, dans sa variante vernaculaire toscane. Ce procédé va de pair avec l'insertion, au fil de la narration, d'une voix étrangère à la diégèse, que l'original français n'a pas. Et celle-ci se manifeste au premier chef par l'usage de l'apostrophe conventionnelle « figuratevi » qui, ressortissant à la transmission d'un récit oral, a la vertu non seulement de faire affleurer dans le discours un narrateur qui le produit, mais encore de susciter l'illusion d'une relation entre celui-ci et le public, qui est ainsi créé dans l'instant même où il est sollicité. Et voilà qui résone comme une petite musique aux oreilles du lecteur de Collodi, qui a souvent rencontré cette marque textuelle dans son œuvre et, notamment, dans Le avventure di Pinocchio. Et cela dès le premier chapitre quand, voulant travailler la bûche qu'il vient de trouver, maestro Ciliegia entend une voix qui lui dit: «Non mi picchiar tanto forte ! ${ }^{21}$. Ce que la narration commente en en appelant à d'hypothétiques allocutaires : " Figuratevi come rimase quel buon vecchio di maestro Ciliegia ${ }^{22}$ (EN III, 65)! » Tout comme dans le théâtre de marionnettes où Pinocchio retrouve ses semblables, dans un monde de poupées de bois gouverné par le terrible Mangiafoco qui, tout comme l'ogre du Petit Poucet, a « un mouton tout entier à la broche pour le souper » (Hachette 1860, 79). Et quand le bois vient à manquer, c'est d'abord Pinocchio que Mangiafoco désigne pour alimenter le

\footnotetext{
${ }^{19}$ Pietro Fanfani-Giuseppe Rigutini. Vocabolario italiano della lingua parlata. Nuovamente compilato da Giuseppe Rigutini. Firenze : Barbèra, 1893.

20 «Ingiuria grave. O fagotto ! Brutto fagotto ! È peggio Fagotto di panni sudici! », Policarpo Petrocchi. Nòvo dizionàrio universale della lingua italiana. Milano : Treves, 1887-1891.

${ }^{21}$ Edizione Nazionale delle opere di Carlo Lorenzini Collodi. Pescia Firenze : Fondazione Nazionale Carlo Collodi-Giunti, 2012. Vol. III. Le avventure di Pinocchio, a cura di Roberto Randaccio, con introduzione di Daniela Marcheschi e prefazione di Mario Vargas Llosa, 65. Désormais cité comme EN III. Version française : « Ne me frappe pas si fort !... » (Carlo Collodi, Les Aventures de Pinocchio, trad. la comtesse de Gencé. Paris : Le Livre de poche, 1990, 44).

22 « Représentez-vous alors l'ébahissement de ce brave père La Cerise (EN III, 44).
} 
feu nécessaire à la cuisson de son mouton ; puis, lui ayant fait grâce, Arlequin lui succède. «Figuratevi il povero Arlecchino ! ${ }^{23}$ précise la narration.

La traduction de Collodi suscite ce procédé dans Le Petit Poucet lorsque « croy[ant] retrouver aisément son chemin par le moyen de son pain qu'il avait semé partout», " il fut bien surpris lorsqu'il ne put en retrouver une seule miette : les oiseaux étaient venus, qui avaient tout mangé » (77) ; ce qu'elle rend par : « figuratevi come rimase, quando si accorse che i minuzzoli glieli avevano beccati gli uccelli » (EN IV , 83). L'apostrophe a une fonction d'intensificateur expressif en même temps qu'elle permet l'irruption d'un narrateur agent du discours. Et cette technique, Collodi l'utilise indifféremment dans les contes des trois auteurs. Dans La Biche au bois de Madame d'Aulnoy, la dame de compagnie de la princesse Désirée, condamnée à se transformer en biche pendant la journée, se trouve nez à nez avec le Prince Guerrier ; et la phrase : " [Giroflée] se hâta d'ouvrir, et demeura bien surprise de voir le prince » (Hachette 1860, 321) est rendue par : « [Viola-a-ciocche] corse subito ad aprire, e figuratevi come restò nel trovarsi a faccia a faccia col Principe »(EN IV, 225). Dans La Belle et la Bête de Madame Leprince de Beaumont, quand, à son réveil dans le château de la Bête, le marchand ruiné découvre un vêtement propre là où il avait déposé ses hardes : «Il était dix heures du matin quand il se leva le lendemain, et il fut bien surpris de trouver un habit fort propre à la place du sien » (Hachette 1860, 354). Ce que Collodi rend de nouveau par : "La mattina dopo si svegliò verso le dieci : e figuratevi come rimase, quando trovò un vestito molto decente nel posto dove aveva lasciato il suo » (EN IV, 244).

Par ce procédé - la création d'une voix narrative extradiégétique comme par d'autres - l'amplification expressive, l'ancrage vernaculaire, le processus de trivialisation -, Collodi tend à uniformiser l'ensemble de l'ouvrage en lui donnant une tonalité unique, quand même il ne renonce pas à restituer les particularités de chaque auteur : mais atténuées, et coulées dans un même creuset où elles se trouvent amalgamées par la force cohésive de la traduction. Et ce qui n'était à l'origine qu'une anthologie, dont la composition avait été dictée par les contingences d'une opération commerciale liée à la Bibliothèque des Chemins de fer, devient, dans sa version italienne, un ouvrage homogène, doté d'une cohérence interne qui ne laisse plus rien paraître de son caractère composite : dans un volume dont il ne reste, pour tout auteur, que le traducteur.

\section{Bibliographie}

${ }^{23}$ « Représentez-vous le pauvre Arlequin !» (EN III, 84.) 
Contes de fées tirés de Claude (sic) Perrault, de Mme d'Aulnoy et de Mme Leprince de Beaumont. Paris : Librairie Hachette, 1860.

Madame d'Aulnoy. Contes des fées. Edition critique établie par Nadine Jasmin. Paris, Honoré Champion, 2008.

Madame d'Aulnoy. Conte nouveaux ou les Fées à la mode. Edition critique établie par Nadine Jasmin. Paris : Honoré Champion, 2008.

Baccini, Ida. La mia vita. Ricordi autobiografici, a cura di Lorenzo Cantatore. Milano : Unicopli, 2004.

Bonanni, Veronica. " "L'Oiseau bleu”" et "L'uccello turchino”. Collodi traducteur d'Aulnoy. " Féeries. Études sur le conte merveilleux (XVIIe -XIXe siècle) 9 (2012) : 251-65.

---. «"I racconti delle fate". Collodi traduttore di Perrault, d'Aulnoy e Leprince de Beaumont. » Quaderno di italianistica 8 (2013) : 93-132.

---. Giannettino. Libro per i ragazzi. Firenze : Bemporad, 1892.

---. La lanterna magica di Giannettino. Libro per i giovanetti. Firenze : Bemporad, 1902.

---. Le avventure di Pinocchio. Edizione critica a cura di Ornella Castellani Pollidori. Pescia : Fondazione Nazionale Carlo Collodi, 1983.

---. Edizione Nazionale delle Opere di Carlo Lorenzini. Pescia Firenze:

Fondazione Nazionale Carlo Collodi-Giunti, 2010. Vol. I. Carlo Lorenzini, Un romanzo in vapore. Da Firenze a Livorno, a cura di Roberto Randaccio, prefazione di Michèle Merger, introduzione di Elvio Guagnini; I Misteri di Firenze, a cura e con introduzione di Roberto Randaccio, prefazione di Andrea Camilleri.

---. Edizione Nazionale delle opere di Carlo Lorenzini Collodi. Pescia Firenze, Fondazione Nazionale Carlo Collodi-Giunti, 2012. Vol. III. Le avventure di Pinocchio, a cura di Roberto Randaccio, con introduzione di Daniela Marcheschi e prefazione di Mario Vargas Llosa.

---. Edizione Nazionale delle opere di Carlo Lorenzini Collodi. Pescia Firenze, Fondazione Nazionale Carlo Collodi-Giunti, 2015. Vol. IV. I racconti delle fate voltati in italiano da Carlo Collodi, a cura e con introduzione di François Bouchard ; Storie allegre, a cura e con introduzione di François Bouchard, prefazione di Guido Conti.

---. I racconti delle fate voltati in italiano da Carlo Collodi, Firenze, Felice Paggi, 1876. 2a edizione, 1887.

---. I racconti delle fate tratti da Perrault, D'Aulnoy e Leprince de Beaumont, versione italiana di Cesare Donati, Firenze, Stefano Jouhaud, 1867.

Parinet, Elisabeth. « Les Bibliothèques de gare, un nouveau réseau pour le livre. » Romantisme 23.80 (1993): 95-106.

Perrault, Charles. Contes. Texte établi, présenté et annoté par Tony Gheeraert. Paris : Honoré Champion, 2012.

Madame de Villeneuve. La Jeune Américaine et les Contes marins (La Belle et la Bête) Les Belles solitaires, avec Madame Leprince de Beaumont, Magasin des enfants (La Belle et la Bête), édition critique établie par Élisa Biancardi. Paris : Honoré Champion, 2008. 